\title{
Simultaneous Optical and Radar Observations of Meteor Head-Echoes utilizing SAAMER
}

\author{
R. G. Michell ${ }^{a, b}$, D. Janches ${ }^{b}$, M. Samara ${ }^{b}$, J. L. Hormaecheac ${ }^{c}$ C. Brunini ${ }^{d}$, \\ I. Bibbo $^{\mathrm{d}}$ \\ ${ }^{a}$ University of Maryland, College Park, College Park, MD, USA \\ (robert.g.michell@nasa.gov001-301-286-5959) \\ ${ }^{b}$ NASA, Goddard Space Flight Center, Greenbelt, MD, USA \\ ${ }^{c}$ Estacion Astronomica Rio Grande, Rio Grande, Tierra del Fuego, Argentina \\ ${ }^{d}$ Departamento de Ciencias Astronomicas y Geofisicas, Universidad Nacional de La \\ Plata, La Plata, Argentina
}

\begin{abstract}
We present simultaneous optical and radar observations of meteors observed with the Southern Argentine Agile MEteor Radar (SAAMER). Although such observations were performed in the past using High Power and Large Aperture radars, the focus here is on meteors that produced head echoes that can be detected by a significantly less sensitive but more accessible radar system. An observational campaign was conducted in August of 2011, where an optical imager was operated near the radar site in Rio Grande, Tierra del Fuego, Argentina. Six head echo events out of 150 total detections were identified where simultaneous optical meteors could also be clearly seen within the main radar beam. The location of the meteors derived from the radar interferometry agreed very well with the optical location, verifying the accuracy of the radar interferometry technique. The meteor speeds and origin directions calculated from the radar data were accurate - compared with the optics - for the 2 meteors that had radar signal-to-noise ratios above 2.5. The optical meteors that produced the head echoes had horizontal velocities in the range of $29 \mathrm{~km} / \mathrm{s}$ to $91 \mathrm{~km} / \mathrm{s}$. These comparisons with optical observations improve the accuracy of the radar detection and analysis techniques, such that, when applied over longer periods of time, will improve the statistics of southern hemisphere meteor observations. Mass estimates were derived using both the optical and radar data and the resulting masses agreed well with each other. All were within an order of magnitude and in most cases, the agreement was within a factor of two.
\end{abstract}

Preprint submitted to Planet. Space Sci.

April 7, 2015

(C) 2015. This manuscript version is made available under the Elsevier user license http://www.elsevier.com/open-access/userlicense/1.0/ 
Keywords: Meteor, Optical, Head Echo, Meteor Mass

\section{Introduction}

The use of combined optical and radar observations of meteors is an under-utilized technique, which can lead to an increased insight into the processes involved with meteor ablation as well as help to constrain key parameters such as the meteor ionization and luminous efficiencies (Weryk and Brown, 2013). The use of High-Power and Large Aperture (HPLA; aka Incoherent Scatter Radars) is currently the primary method for observing the small signals from meteor head echoes, but recently Janches et al. (2014a) demonstrated that all-sky VHF meteor radars with advanced designs such as the Southern Argentine Agile MEteor Radar (SAAMER) (Fritts et al., 2010), can also be used to detect such events. SAAMER transmits significantly less power compared to HPLA systems but 5 to 10 times more power than similar all-sky VHF meteor radars, which typically detect specular meteor trail echoes - a much larger scattering target than head-echoes. The use of a transmitting antenna array that can focus the transmitted power is also essential to detect the head echoes.

The analysis and modeling of meteor head echoes in HPLA radar observations has been used to constrain the characteristics of the incoming meteoroid flux (Mathews et al., 2001; Close et al., 2005; Dyrud and Janches, 2008; Fentzke and Janches, 2008; Close et al., 2012; Janches et al., 2014b). The utilization of HPLA systems to detect meteor head echoes is currently the most efficient way to detect and quantify large numbers of meteors, especially the most abundant, small, microgram to milligram interplanetary Dust Particles (IDP). HPLA observations of meteor head echoes can provide accurate measurements of only a limited number of meteor parameters, including the line-of-sight (LOS) velocity and deceleration as well as the backscattered power. The absolute velocity can only be determined for those systems that have interferometric capabilities (Chau and Woodman, 2004; Sparks et al., 2010; Kero et al., 2011; Pifko et al., 2013). It is from these fairly limited measurements that other parameters, such as mass can be estimated with less accuracy.

The study of meteors and their interactions with planetary atmospheres involve many aspects, including theory and modeling, such as described in McNeil (1999); Oppenheim et al. (2000); Dyrud et al. (2007); Close et al. 
(2002) and more recently the techniques described in Gritsevich (2009); Bouquet et al. (2014); Moreno-Ibáñez et al. (2015) to name a few representative examples. In addition, there have been many observational studies which use radars (Reddi and Nair, 1998; Janches et al., 2003), lidars (Grime et al., 1999; Von Zahn, 2001), and spectroscopy and imaging (Shamir, 2005; Kaiser et al., 2004). However, there appears to be a lack of studies that combine optical television observations of visible meteor trails with simultaneous radar scattering signatures, until very recently (Michell, 2010; Campbell-Brown et al., 2012; Weryk and Brown, 2013). In the last decade, the availability of Electron Multiplying CCD (EMCCD) imagers have greatly increased the ease of analyzing high-time resolution, low-light-level optical imaging. Most of the observational work on radar scattering from meteors is done without optical imaging, commonly assuming that the meteors causing the radar scattering are too small to produce visible trails. Initial observations - using the Poker Flat Incoherent Scatter Radar (PFISR) - demonstrated that meteors detected optically were simultaneously observed as head echoes by the radar, even when they occurred in the side lobes (Michell, 2010). Additionallyfor the case of PFISR observations - there appears to be a direct correlation between optical brightness and radar backscattered power-radar cross section (RCS) - indicating a mass dependence of the RCS (Michell, 2010; Close et al., 2012).

The focus of the observations presented here is on common-volume optical imaging and SAAMER observations of meteor head echoes, where we have identified - optically - some of the meteors that produced the head echoes in the radar data. The main purpose of these comparisons is to demonstrate that such observations can be done with more accessible and less expensive systems than HPLA radars. In addition, the added information from the optical observations is used to improve the accuracy of the radar detection and analysis techniques. Therefore the application of such radar observations over longer periods of time - when optical data are not available or not possible due to daylight and weather conditions - enables the compilation of more accurate statistics of southern hemisphere meteor observations. In the next section, the methods are described, including both optical and radar. The observations are then presented, followed by discussion and conclusions. 


\section{Methodolgy}

The data presented in this work were obtained over a period of 12 days covering 02 to 14 August, 2011. Details of the observing campaign are described in Janches et al. (2014a). Due to the low sensitivity of SAAMER, we did not expect meteor head-echo detection rates to be as large as is the case for HPLA radars (> 10 events/minutes at the peak). In addition, because these observations were performed simultaneously with the optical imager, we concentrated mostly on night hours, with the inclusion of mornings to cover the flux rate increase and peaks (Janches et al., 2006), thus increasing the likelihood of successful observations.

\subsection{Radar Observations}

SAAMER is a SKiYMET system (Hocking et al., 1997) deployed at the Estacion Astronomica Rio Grande (EARG) in the city of Rio Grande (53.8 $45^{\prime} 8^{\prime \prime}$ S; $67^{\circ} 45^{\prime} 5$ " W), province of Tierra del Fuego, Argentina. The system, which operates at a frequency of $32.55 \mathrm{MHz}$, is enhanced relative to standard meteor radars, including higher transmitter peak power $-60 \mathrm{~kW}$, rather than 6-20 kW used by most meteor radar systems (Hocking et al., 2001; Brown et al., 2008; Stober et al., 2011) — and a transmitter phase antenna array configuration, specially designed by Mardoc Inc., composed of eight 3-element crossed yagis arranged in an octagon of $27.6 \mathrm{~m}$ (3 wavelengths) in diameter. This is significantly different from typical systems, which use a single antenna. This provides the ability to change electronically (e.g. pulse to pulse) the phases between antennas allowing transmission of different radiation patterns and hence permits the operation of a number of different experiments (Janches et al., 2014a). During the observing campaign reported here, we utilized a transmitting mode that somewhat follows the methodology applied in the past for meteor head echo observations utilizing HPLA radars by transmitting with all the TX antennas in phase resulting in most of the radiated power upwards in a relatively narrow beam. As described in Janches et al. (2014a), this methodology results in a near Gaussian central transmitted beam pattern with a $3 \mathrm{~dB}$ decrease in gain at $\sim 8^{\circ}$. We refer to this mode as a "relatively" narrow beam because when compared with HPLA systems, SAAMER's main beam width is approximately 3 times wider than the MU and ALTAIR radars (Close et al., 2000; Kero et al., 2011), 8 times wider than PFISR and Jicamarca (Chau and Woodman, 2004; Sparks et al., 2010) and 


\begin{tabular}{lr}
\hline \hline Quantity (units) & $53.8^{\circ} \mathrm{S}$ \\
\hline \hline Latitude (degrees) & $67^{\circ} \mathrm{W}$ \\
Longitude (degrees) & 32.55 \\
Frequency (MHz) & 500 \\
PRF (Hz) & 60 \\
Peak Transmitted Power (kW) & 0.05 \\
Banwidth (MHz) & 2 \\
Coherent Integrations (\# of IPP) & Monopulse \\
Pulse Code & 13.6 \\
Pulse Length ( $\mu$ s) & 250 \\
Sampling Resolution $(\mathrm{m})$ & $8^{\circ}$ \\
FWHM &
\end{tabular}

Table 1: SAAMER's operating characteristics for the head-echo mode

50 times wider than the Arecibo radar (Janches et al., 2004), yet is significantly ( 18 times) narrower than the typical all-sky pattern resulting from a single Yagi antenna utilized in most of the meteor radar systems (Fritts et al., 2012). Specifically, we transmitted a $13.5 \mu$ s monopulse at a Pulse Repetition Frequency (PRF) of $500 \mathrm{~Hz}$ and performed a 2 point pulse coherent integration, thus resulting in an effective Inter Pulse Period (IPP) of $4 \mathrm{~ms}$. The sampling rate of the returned signal results in $250 \mathrm{~m}$ range resolution and the bandwidth was $0.05 \mathrm{MHz}$. The vertical altitude range covered was between $\sim 75 \mathrm{~km}$ and $130 \mathrm{~km}$. Table 1 presents a summary of SAAMER's operation characteristics. This scheme was chosen as an initial test mode, where the data rate could be handled by the current computer. The larger area and lower transmitted power, as compared to most HPLA systems, results in lower power density and hence sensitivity to larger particles than those detected by typical HPLA radars. Therefore using the head echo observing mode with SAAMER, the size range of meteoroid observations is extended to larger masses as compared to HPLA radars, with the exception of the MU radar in Japan (Kero et al., 2011) which can also detect meteoroids of a similar size range as SAAMER.

Table 2 summarizes the orbital parameters of the six meteors reported on here. These are the semi-major axis $(a)$ in AU, the eccentricity $(\epsilon)$, the inclination $(I)$, the ecliptic longitude $(\lambda)$ and the ecliptic latitude $(\beta)$. It 


\begin{tabular}{|c|c|c|c|c|c|}
\hline Meteor \# & $a(\mathrm{AU})$ & $\epsilon$ & $I$ (Deg.) & $\lambda$ (Deg.) & $\beta$ (Deg.) \\
\hline \hline 1 & 1.23 & 0.32 & 168 & -8.4 & -6.3 \\
\hline 2 & 2.6 & 0.83 & 101.9 & -37.7 & -30.7 \\
\hline 3 & 1.8 & 1.5 & 153.4 & 7.52 & -16.46 \\
\hline 4 & 1.3 & 0.3 & 96.3 & -9.6 & -44.1 \\
\hline 5 & 10.8 & 0.9 & 107.7 & 4.1 & -43 \\
\hline 6 & 0.6 & 0.8 & 91.7 & 20.4 & -21.9 \\
\hline
\end{tabular}

Table 2: Summary of the orbital parameters of the meteors, derived from the radar measurements. These include the semi-major axis $(a)$ in AU, the eccentricity $(\epsilon)$, the inclination $(I)$, the ecliptic longitude $(\lambda)$ and the ecliptic latitude $(\beta)$. Note, the ecliptic longitude $(\lambda)$ is measured from the apex.

should be noted that for the data presented in Table 2, the ecliptic longitude $(\lambda)$ is measured from the apex, thus Earth's apex is $0^{\circ}$ with the sun at $90^{\circ}$.

\subsection{Optical Observations}

One imager from the Multi-spectral Observatory Of Sensitive EMCCDs (MOOSE) suite of imagers was used to make the observations. These are Andor Ixon DU-888 EMCCD imagers that have a $1024 \times 1024$ pixel chip, with internal binning and sub-framing capabilities that allow tradeoffs between temporal and spatial resolution. For the observations presented here, the imager was operating with a narrow field of view $\left(14^{\circ}\right)$. The CCD was internally cooled to $-70^{\circ} \mathrm{C}$ to reduce thermal noise and was set to $4 \times 4$ binning with some sub-framing, resulting in a $190 \times 190$ image at 40.7 frames per second ( $\sim 20 \mathrm{~ms}$ exposure time). This setup provides an angular resolution of $0.073^{\circ}$ per pixel or approximately 130 meters per pixel, assuming an altitude for the meteors of $\sim 100 \mathrm{~km}$. The imager was pointed at the local zenith $\left(90^{\circ} \mathrm{El}\right.$.) and was located approximately $5 \mathrm{~km}$ from the radar transmitter in order to avoid the bright lights of the city and the airport. The center of the radar beam was therefore offset by approximately $5 \mathrm{~km}$ from the center of the image.

\section{Observations}

During the near two-week campaign the radar detected about 150 meteor head echoes (Janches et al., 2014a). Unfortunately, during this period there 


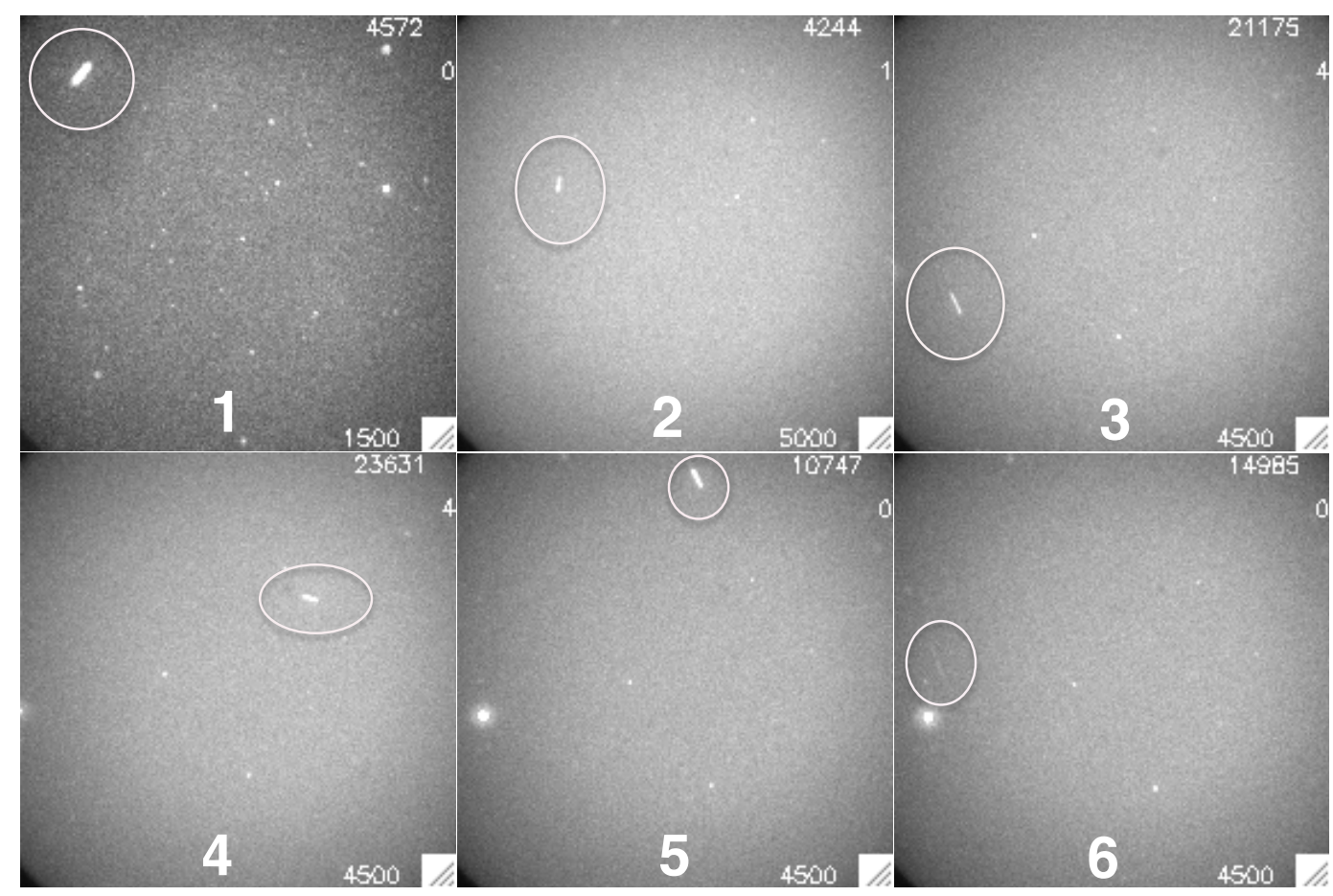

Figure 1: Images showing the six meteors. The meteors are circled and the white number is the meteor number for reference. The area of the images is approximately $25 \mathrm{~km} \mathrm{x} 25$ $\mathrm{km}$, at $100 \mathrm{~km}$ altitude.

were few nights with optimal weather for the optical observations. Nevertheless, cross-referencing the times when it was clear enough for the imager to detect optical meteors, a total of six of the radar detected meteors were also detected with the imager.

Figure 1 shows a panel of optical images for each of the six meteors simultaneously detected by both techniques, the meteors are circled for identification and numbered 1-6. Meteor \#6 was traveling faster than the rest and therefore has a longer, fainter streak, due to the finite exposure time of $\sim 20 \mathrm{~ms}$, but is clearly identifiable in 7 frames as it passes through the image. Figure 2 shows a panel with the corresponding radar data as RangeTime-Intensity (RTI) images for each of these six meteors.

SAAMER used - as a receiver - the typical 5 antenna interferometer arrangement which allowed for the unambiguous determination of the spatial location of the detected echoes. This methodology is widely utilized and will not be described in this work. Hocking et al. (1997) and Hocking et al. (2001) described in detail the operation of the 5 antenna meteor radar inter- 

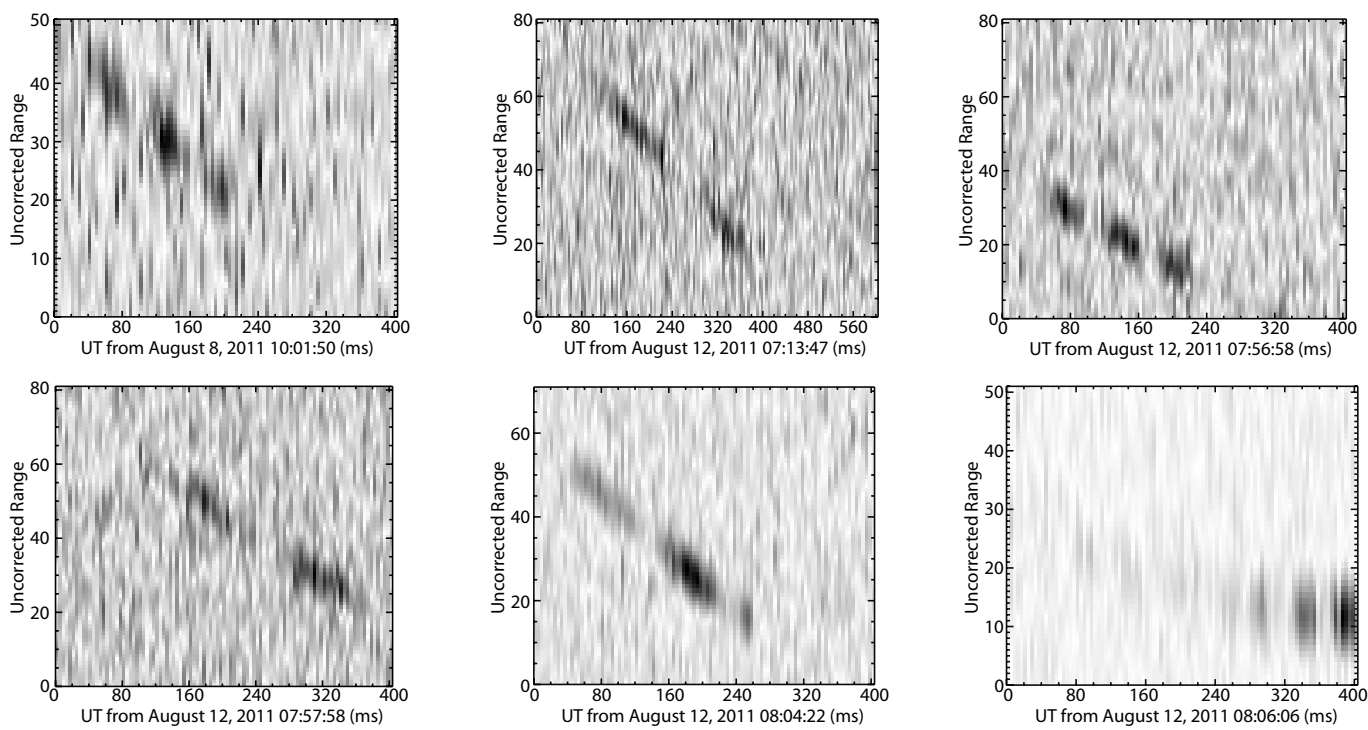

Figure 2: Radar returns from the six meteors, plotted as Range-Time-Intensity plots. The numbering is in the same orientation as is in Figure 1.

ferometer. The application of interferometry for meteor head echo purposes is described in Janches et al. (2014a). The positions and velocities of the meteors can therefore be calculated from the radar observations. The radar derived meteor speeds and directions can then be compared with the speeds and directions determined from the optical observations. Figure 3 shows a panel of optical images, showing the meteors' locations from the images (white boxes) and the locations derived from the radar data (black stars). The white ' $\mathrm{X}$ ' in the upper left corner denotes the position of the center of the radar beam. The small white arrows indicate the direction of travel of the optical meteors. The radar data are the positions of the meteors that were derived from the interferometry from different times along their paths.

The horizontal speeds and the directions of origin for these meteors can be derived from both the radar and optical data independently. Table 3 summarizes the comparison between the optical and radar derived horizontal speeds ( \pm estimated errors) and origin directions for these 6 meteors.

\section{Discussion}

The meteor head echo observations with SAAMER presented in (Janches et al., 2014a) represent a great improvement to our meteor observing capabilities in the southern hemisphere. The observations presented here build 


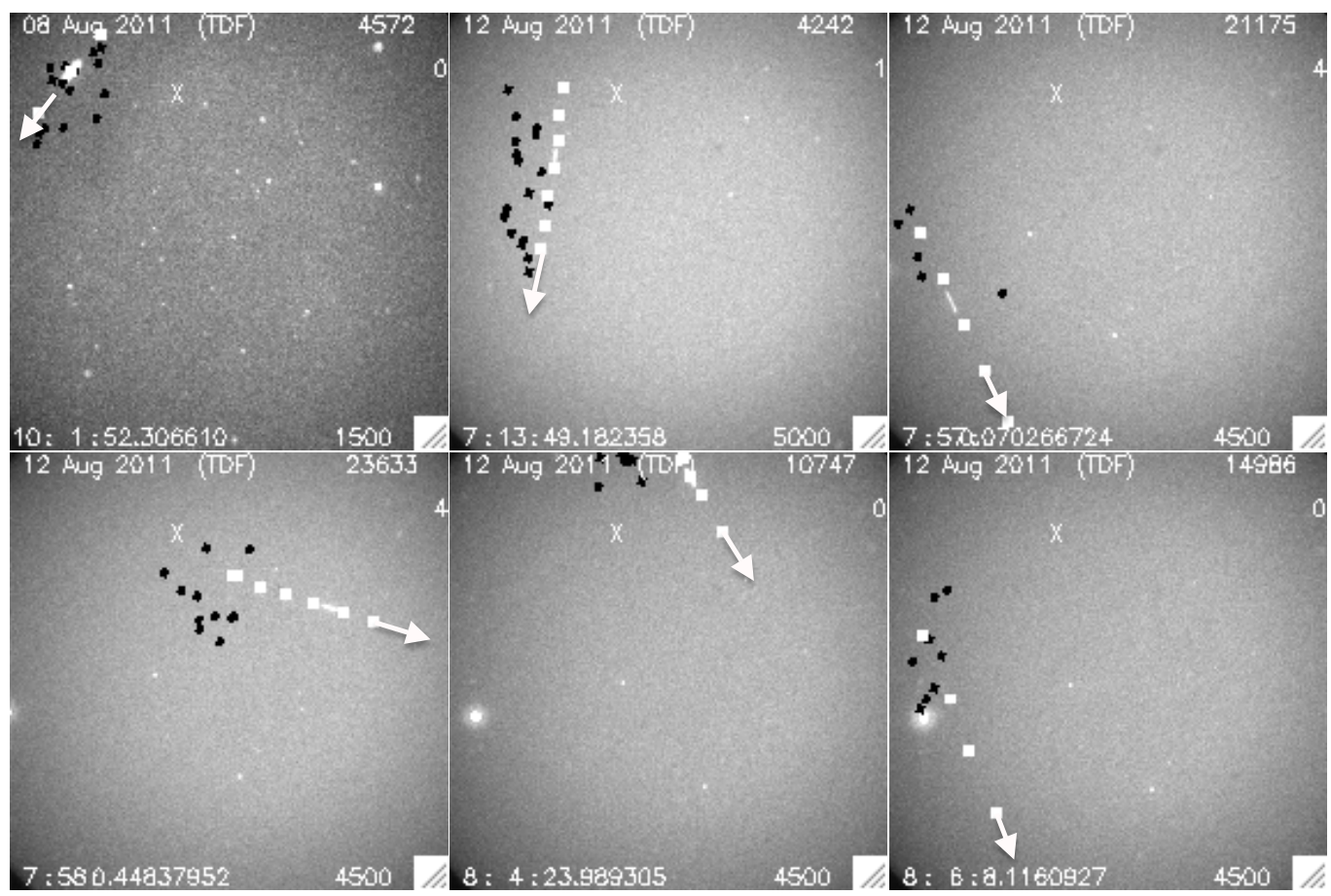

Figure 3: Images showing the optical meteor locations (white boxes) and the locations derived from the radar interferometry (black stars). The white ' $\mathrm{X}$ ' in the upper left corner of the images marks the location directly above the radar (the position of the center of the radar beam).

\begin{tabular}{|c|c|c||c|c|}
\hline $\begin{array}{c}\text { Meteor } \\
\#\end{array}$ & $\begin{array}{c}\text { Optical } \perp \\
\text { Speed }(\mathrm{km} / \mathrm{s})\end{array}$ & $\begin{array}{c}\text { Radar } \perp \\
\text { Speed }(\mathrm{km} / \mathrm{s})\end{array}$ & $\begin{array}{c}\text { Optical } \\
\text { Dir. (Deg.) }\end{array}$ & $\begin{array}{c}\text { Radar } \\
\text { Dir. (Deg.) }\end{array}$ \\
\hline \hline 1 & $57.2( \pm 5.1)$ & $53.7( \pm 13.2)$ & $329.8^{\circ}$ & $357.9^{\circ}$ \\
\hline 2 & $29.5( \pm 1.7)$ & $30.1( \pm 7.3)$ & $18.2^{\circ}$ & $36.9^{\circ}$ \\
\hline 3 & $75.4( \pm 2.5)$ & $67.6( \pm 8.6)$ & $58.7^{\circ}$ & $60.4^{\circ}$ \\
\hline 4 & $52.0( \pm 2.0)$ & $34.3( \pm 10.4)$ & $90.5^{\circ}$ & $52.3^{\circ}$ \\
\hline 5 & $41.2( \pm 3.4)$ & $42.9( \pm 10.2)$ & $61.0^{\circ}$ & $69.0^{\circ}$ \\
\hline 6 & $91.5( \pm 3.4)$ & $62.3( \pm 4.6)$ & $56.9^{\circ}$ & $41.1^{\circ}$ \\
\hline
\end{tabular}

Table 3: Summary of the radar and optical comparison of speeds and directions for the meteors. The estimated errors in the speeds are listed in the parentheses (in $\mathrm{km} / \mathrm{s}$ ). The direction is defined as the direction of origin of the meteor with North $=0^{\circ}$ and East $=$ $90^{\circ}$. 
on that analysis, with the goal of improving the meteor detection and analysis techniques using the radar data alone. Optical observations of the same meteors detected in the radar can be used to accurately determine the direction of motion of the meteors and - given the high frame rate of the imager data - the horizontal component of the velocity.

The summary of the optical and radar data presented in Figure 3 shows that agreement is found in the radar and optical observations, suggesting that these are simultaneous radar and optical detections of the same events. However, there is significant spread in the radar derived locations. In addition, the meteors appear to have more radar position determinations along the portion of their paths that are the closest to the center of the radar beam, marked by the white ' $\mathrm{X}$ ' in Figure 3. This is consistent with the beam pattern of the radar during these observations, which had a single beam with a $3 \mathrm{~dB}$ point at $8^{\circ}$ off zenith. The size of the images is $14^{\circ} \times 14^{\circ}$, meaning that most of the radar derived points were within about $5^{\circ}$ of the beam center, emphasizing the need for focused transmitted power in order to be able to make head-echo detections (Janches et al., 2014a). The scattered nature of these points indicates that there was not always enough signal in the radar to determine a location, indicating that the data are noisy and the technique utilized in this campaign has limitations.

The comparison between the directions and speeds determined from the radar and optical data are useful in evaluating the accuracy and limitationsas well as methods for improving - the radar data analysis techniques. Table 4 summarizes the differences between the optical and radar horizontal speeds and directions. The radar speed estimates overall appear to be slightly more accurate than the direction estimates. Four out of the six speeds differ by less than $10 \mathrm{~km} / \mathrm{s}$ (two of them less than $2 \mathrm{~km} / \mathrm{s}$ difference), while the for the directions, only 2 of them differ by less than 10 degrees.

Meteors 1, 2, 3 and 5 all had speeds that closely matched between the radar and optics, while meteors 3 and 5 had origin directions that matched fairly closely. Meteor 4 and meteor 6 had significant differences in both speed and direction between the optics and radar. Although the returns for these meteors were centered close to the center of the radar beam, the spread was significant about the meteor path, producing the larger errors.

The optically measured speeds tend to be more accurate because the 2-dimensional position of the meteor can be located to within two pixels in multiple frames (between 4 and 13 different frames for these meteors), leading to maximum errors in speeds of between 1 and $5 \mathrm{~km} / \mathrm{s}$. The radar 


\begin{tabular}{|c|c|c||c|}
\hline $\begin{array}{c}\text { Meteor } \\
\#\end{array}$ & $\begin{array}{c}\perp \text { Speed } \\
\text { Diff. }(\mathrm{km} / \mathrm{s})\end{array}$ & $\begin{array}{c}\text { Direction } \\
\text { Diff. (Deg.) }\end{array}$ & $\begin{array}{c}\text { Radar } \\
\text { SNR }\end{array}$ \\
\hline \hline 1 & 3.5 & $-28.1^{\circ}$ & 0.7 \\
\hline 2 & -0.6 & $-18.7^{\circ}$ & 1.9 \\
\hline 3 & 7.8 & $-1.7^{\circ}$ & 2.5 \\
\hline 4 & 17.7 & $38.2^{\circ}$ & 2.1 \\
\hline 5 & -1.7 & $-8.0^{\circ}$ & 2.7 \\
\hline 6 & 29.2 & $15.8^{\circ}$ & 1.6 \\
\hline
\end{tabular}

Table 4: The differences between the optical and radar horizontal speeds and origin directions as well as the radar signal level for the meteors, expressed as the signal-to-noise ratio.

data however have errors in the 5 to $10 \mathrm{~km} / \mathrm{s}$ range.

The estimated errors in the meteor origin directions calculated from the optical data range between $2^{\circ}$ and $5^{\circ}$, while the estimates based on the radar data have errors that range between $10^{\circ}$ and $15^{\circ}$. The calculation of the meteor origin directions show more variation between the optics and radar than the perpendicular speeds do. This indicates that the radar method of calculating directions appears to be sensitive to the errors introduced by the low signal levels - from either weak backscatter or the meteor being too far from the center of the radar beam.

The data displayed in Figure 3 show that the radar interferometry technique accurately captures the spatial location of the meteors on average. There is some spread in the radar derived locations about the actual meteor location, which causes the errors in the speed and direction calculations. Two of the meteors (3 and 5) showed fairly good agreement between both the optically derived and radar derived perpendicular speeds and directions. These events have the highest radar Signal-to-Noise Ratios (SNR) of all the meteors, 2.5 and 2.7, respectively. Two of the other meteors (1 and 2 ) showed agreement between the optics and radar in only one of the parameters (speed). The remaining two meteors (4 and 6) showed large differences between the radar and optically derived speeds and directions. The radar head echo observations analyzed here actually represent some of the weakest backscattered power head echo events detected during the campaign. These six meteors are the only head echoes detected when the observing conditions were favorable for the optical observations. In fact, many other head 
echoes - with much stronger backscattered power - were detected at different times with the radar and these had much smaller errors in the direction and velocity estimations (Janches et al., 2014a), but the sky conditions at those times were unfavorable for optical observations - either cloudy or snowing.

It is important to note that, in order to make these observations, the radar was operated in a different mode than its normal operational design, which is for determining mesospheric winds estimates. In light of this and the fact that most of these meteors represent the weakest head echo events detected, these observations show that it is possible to calculate meteor speeds and directions from meteor head echoes detected by the radar. In fact, this method produces accurate estimates of the meteor speeds and directions for meteors with radar SNR values above about 2.5. SAAMER - operated in this mode - can detect about one head echo per hour with SNR greater than 2.5 , and if these simultaneous observations can be performed continuously it will enable the collection of sufficient statistics of these relatively rare events.

The mass of meteors in general can be estimated from both optical and radar methods (Gritsevich, 2009; Campbell-Brown et al., 2012; Weryk and Brown, 2013), each of which relies on separate assumptions. The mass of the meteors presented here, was estimated from both the optical and radar observations, using independent techniques. The summary of the mass estimates is presented in Table 5. The optical mass was calculated using a luminous efficiency of 1\%, consistent with Hill et al. (2005) and a radiative energy output constant of $1950 \mathrm{~W}$, consistent with Ceplecha et al. (1998). The radar masses were derived using a recently developed model reported in Janches et al. (2014b). The model utilized the Chemical Ablation Model (CABMOD) developed by Vondrak et al. (2008) to calculate the electron production profiles as a function of altitude of a given particle, and the radar antenna gain pattern to estimate the meteor SNR as a function of the physical location of the particle's path through the radar beam. The velocity and spatial information derived from the SAAMER observations are input back into the model in order to estimate the mass of the particle required to produced the detected signal. The possible mass ranges derived for each meteor are presented in Table 5 .

Figure 4 shows a plot of the optical masses of the meteors plotted against the maximum radar SNR. The mass estimates range between $0.1 \mathrm{mg}$ and $1.5 \mathrm{mg}$, which is consistent with the mass range determined by the radar sensitivity estimates presented in Janches et al. (2014a). The data show a general linear relationship, except for meteor \#1 which has a low SNR and 


\begin{tabular}{|c|c|c|c|c|c|}
\hline $\begin{array}{c}\text { Meteor } \\
\#\end{array}$ & $\begin{array}{c}\text { Radar } \\
\text { SNR }\end{array}$ & $\begin{array}{c}\text { Speed } \\
(\mathrm{km} / \mathrm{s})\end{array}$ & $\begin{array}{c}\text { Optical } \\
\text { Mass }(\mathrm{mg})\end{array}$ & $\begin{array}{c}\text { Radar } \\
\text { Mass Range }(\mathrm{mg})\end{array}$ & $\begin{array}{c}\text { Min. } \\
\text { Mag. }\end{array}$ \\
\hline \hline 1 & 0.7 & 64.3 & 1.46 & $0.3-0.5$ & 2.66 \\
\hline 2 & 1.9 & 52.0 & 0.90 & $0.3-0.7$ & 4.20 \\
\hline 3 & 2.5 & 80.0 & 0.88 & $0.6-0.8$ & 3.50 \\
\hline 4 & 2.1 & 63.7 & 0.64 & $0.2-0.6$ & 3.63 \\
\hline 5 & 2.7 & 57.1 & 1.10 & $0.2-0.3$ & 2.82 \\
\hline 6 & 1.6 & 92.3 & 0.10 & $0.5-0.6$ & 5.01 \\
\hline
\end{tabular}

Table 5: The total speeds, mass estimates and minimum magnitudes (maximum brightness) for each of the meteors.

a large optical mass. The correlation coefficient of the data where the SNR is greater than 1.0 (5 data points) is 0.83 , while the correlation coefficient of the data where the SNR is greater than 2.0 (3 data points) is 0.98 . While a definitive relationship between optical mass and radar SNR is not possible with such a small sample size, these initial observations suggest that a relationship exists between these two parameters. Therefore, if this relationship can be quantified with further observations, it can provide a calibration for the radar observations to produce improved meteor mass estimates.

\section{Conclusions}

The use of a relatively inexpensive and accessible radar system - such as SAAMER - to detect meteor head echoes in the southern hemisphere, presents a great advantage over HPLA radars in that such a system can be operated continuously over long periods of time, while HPLA radars can only be operated in meteor modes for short campaigns. Such long term operations allow for the compilation of large statistical samples of meteor head echoes as well as capture the seasonal variations in the meteor populations.

These observations show that this fairly low power SKiYMET radar system can indeed detect meteor head echoes and that the interferometry technique is accurate at determining the physical locations of the meteors. The comparison between the horizontal speeds and directions calculated using both the optical and radar observations reveals that a radar SNR of 2.5 is the lower limit for producing accurate estimates from the radar data.

Two of the six meteors showed good agreement between the radar and 


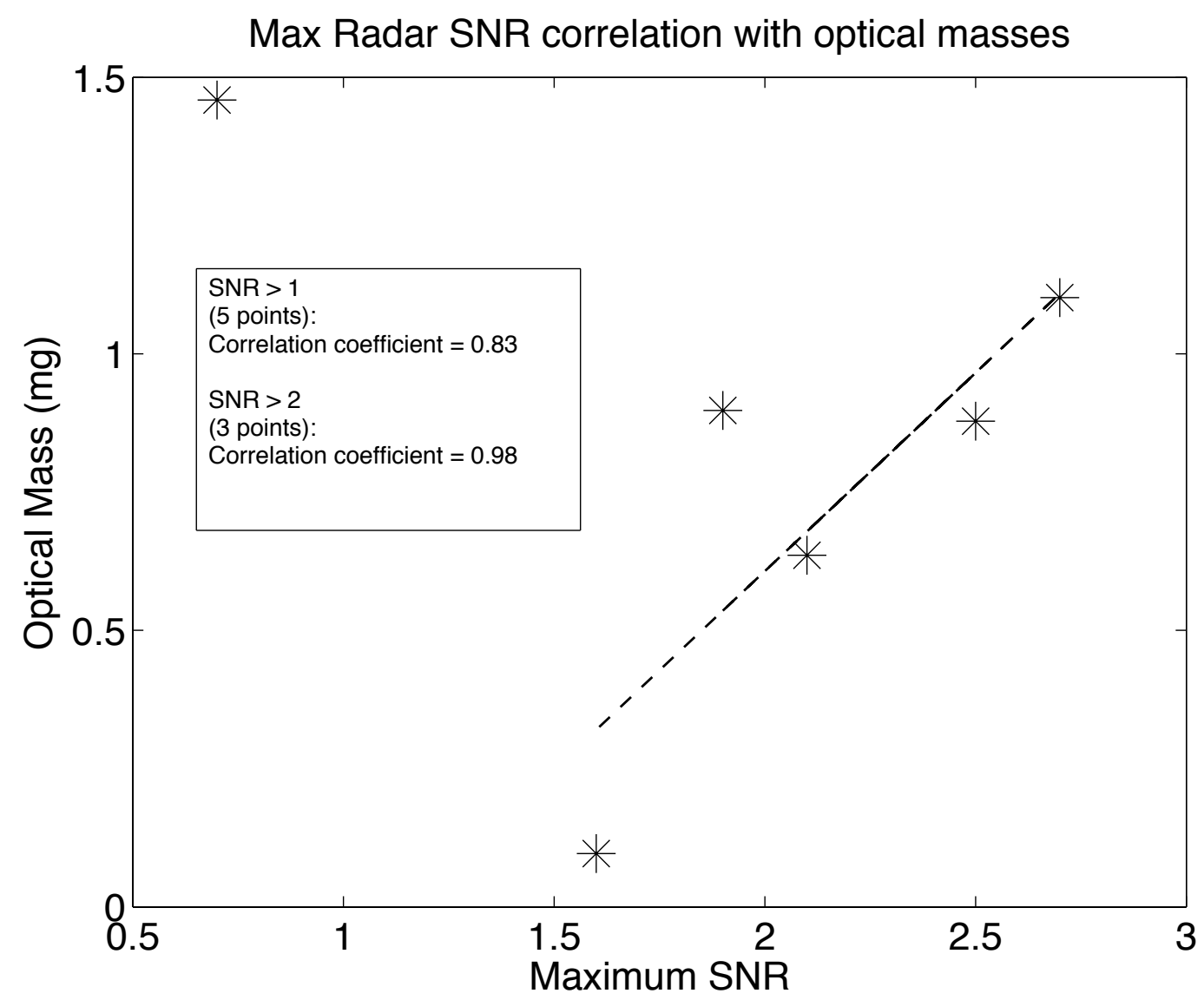

Figure 4: Plot of optical mass versus maximum radar SNR. The dashed line is a fit to the data where the radar SNR is greater than 1.0. 
optics for both the horizontal speed $(<10 \mathrm{~km} / \mathrm{s})$ and direction $\left(<10^{\circ}\right)$ of the meteor, these had the highest radar SNR values (2.5 and 2.7). The meteors with radar SNR less than 2.5 showed either close agreement for the horizontal speed $(<5 \mathrm{~km} / \mathrm{s})$, but not good agreement on the direction $\left(<30^{\circ}\right)$ or large discrepancies in both speed and direction.

The interferometry technique used at this radar is accurate at quantifying meteor parameters and has the potential to be used as a method of determining the speeds and directions of meteors in the hundred microgram to several milligram mass range, which can then be used to calculate the original orbits of the meteors. The comparison to optical observations presented here suggests that one way to ensure the accuracy of the radar determined parameters is to use a threshold backscattered power, under which, it is known that the technique produces larger errors, but over which, it can be shown that the technique produces accurate estimates of the meteor parameters. The comparison presented here provides an initial estimate of the limiting threshold of the radar interferometry technique (radar SNR > 2.5), but further optical observations would be needed in order to statistically quantify the relation between radar accuracy, number of data points, returned power and meteor mass. The optical and radar derived mass estimates agreed well with each other; all within and order of magnitude and in most cases, the agreement was within a factor of two.

\section{Appendix A. Acknowledgements}

The research was funded in part by Southwest Research Institute internal research and development grant. RM is supported by NSF grants AGS1456161 and AGS-1456129. SAAMER is supported by NSF awards AGS0944104 and AST-0908118. DJ is supported by NASA awards 12-PAST120007 and 12-PATM12-0006. The authors would like to thank Jonathan Yee and Stephen Pifko for their assistance in the observational campaign as well as all the staff at Estacion Astronomica Rio Grande for their generosity and hospitality. We thank N. Swarnalingam for his assistance in determining the radar derived masses.

\section{References}

Bouquet, A., Baratoux, D., Vaubaillon, J., Gritsevich, M. I., Mimoun, D., Mousis, O., Bouley, S., Nov. 2014. Simulation of the capabilities of an 
orbiter for monitoring the entry of interplanetary matter into the terrestrial atmosphere. Planet. Space Sci. 103, 238-249.

Brown, P., Weryk, R. J., Wong, D. K., Jones, J., May 2008. A meteoroid stream survey using the Canadian Meteor Orbit Radar. I. Methodology and radiant catalogue. ICARUS 195, 317-339.

Campbell-Brown, M. D., Kero, J., Szasz, C., Pellinen-Wannberg, A., Weryk, R. J., Sep. 2012. Photometric and ionization masses of meteors with simultaneous EISCAT UHF radar and intensified video observations. Journal of Geophysical Research (Space Physics) 117, 9323.

Ceplecha, Z., Borovička, J., Elford, W. G., Revelle, D. O., Hawkes, R. L., Porubčan, V., Šimek, M., Sep. 1998. Meteor Phenomena and Bodies. Space Science Review 84, 327-471.

Chau, J. L., Woodman, R. F., Mar. 2004. Observations of meteor-head echoes using the Jicamarca $50 \mathrm{MHz}$ radar in interferometer mode. Atmospheric Chemistry \& Physics 4, 511-521.

Close, S., Hunt, S. M., Minardi, M. J., McKeen, F. M., Sep. 2000. Analysis of Perseid meteor head echo data collected using the Advanced Research Projects Agency Long-Range Tracking and Instrumentation Radar (ALTAIR). Radio Science 35, 1233-1240.

Close, S., Oppenheim, M., Durand, D., Dyrud, L., Sep. 2005. A new method for determining meteoroid mass from head echo data. J. Geophys. Res. 110,9308 .

Close, S., Oppenheim, M., Hunt, S., Dyrud, L., Oct. 2002. Scattering characteristics of high-resolution meteor head echoes detected at multiple frequencies. J. Geophys. Res. 107, 1295.

Close, S., Volz, R., Loveland, R., Macdonell, A., Colestock, P., Linscott, I., Oppenheim, M., Sep. 2012. Determining meteoroid bulk densities using a plasma scattering model with high-power large-aperture radar data. ICARUS 221, 300-309.

Dyrud, L., Janches, D., Sep. 2008. Modeling the meteor head echo using Arecibo radar observations. Journal of Atmospheric and Solar-Terrestrial Physics 70, 1621-1632. 
Dyrud, L. P., Kudeki, E., Oppenheim, M., Dec. 2007. Modeling long duration meteor trails. J. Geophys. Res. 112, 12307.

Fentzke, J. T., Janches, D., Mar. 2008. A semi-empirical model of the contribution from sporadic meteoroid sources on the meteor input function in the MLT observed at Arecibo. Journal of Geophysical Research (Space Physics) 113, 3304 .

Fritts, D. C., Janches, D., Hocking, W. K., Oct. 2010. Southern Argentina Agile Meteor Radar: Initial assessment of gravity wave momentum fluxes. Journal of Geophysical Research (Atmospheres) 115, 19123.

Fritts, D. C., Janches, D., Hocking, W. K., Mitchell, N. J., Taylor, M. J., May 2012. Assessment of gravity wave momentum flux measurement capabilities by meteor radars having different transmitter power and antenna configurations. Journal of Geophysical Research (Atmospheres) 117, 10108.

Grime, B. W., Kane, T. J., Collins, S. C., Kelley, M. C., Kruschwitz, C. A., Friedman, J. S., Tepley, C. A., Mar. 1999. Meteor trail advection and dispersion; preliminary lidar observations. Geophys. Res. Lett. 26, 675678.

Gritsevich, M. I., Aug. 2009. Determination of parameters of meteor bodies based on flight observational data. Advances in Space Research 44, 323334 .

Hill, K. A., Rogers, L. A., Hawkes, R. L., Dec. 2005. High geocentric velocity meteor ablation. Astronomy and Astrophysics 444, 615-624.

Hocking, W. K., Fuller, B., Vandepeer, B., Jan. 2001. Real-time determination of meteor-related parameters utilizing modern digital technology. Journal of Atmospheric and Solar-Terrestrial Physics 63, 155-169.

Hocking, W. K., Thayaparan, T., Jones, J., 1997. Meteor decay times and their use in determining a diagnostic mesospheric Temperature-pressure parameter: Methodology and one year of data. Geophys. Res. Lett. 24, $2977-2980$.

Janches, D., Heinselman, C. J., Chau, J. L., Chandran, A., Woodman, R., Jul. 2006. Modeling the global micrometeor input function in the upper 
atmosphere observed by high power and large aperture radars. J. Geophys. Res. 111, 7317.

Janches, D., Hocking, W., Pifko, S., Hormaechea, J. L., Fritts, D. C., Brunini, C., Michell, R., Samara, M., 2014a. Interferometric meteor head echo observations using the Southern Argentina Agile Meteor Radar. J. Geophys. Res. 119.

Janches, D., Nolan, M. C., Meisel, D. D., Mathews, J. D., Zhou, Q. H., Moser, D. E., Jun. 2003. On the geocentric micrometeor velocity distribution. J. Geophys. Res. 108, 1222.

Janches, D., Nolan, M. C., Sulzer, M., Apr. 2004. Radiant measurement accuracy of micrometeors detected by the Arecibo $430 \mathrm{MHz}$ Dual-Beam Radar. Atmospheric Chemistry \& Physics 4, 621-626.

Janches, D., Plane, J. M. C., Nesvorný, D., Feng, W., Vokrouhlický, D., Nicolls, M. J., Nov. 2014b. Radar Detectability Studies of Slow and Small Zodiacal Dust Cloud Particles. I. The Case of Arecibo $430 \mathrm{MHz}$ Meteor Head Echo Observations. The Astrophysical Journal 796, 41.

Kaiser, N., Brown, P., Hawkes, R. L., Dec. 2004. Optical Trail Width Measurements of Faint Meteors. Earth Moon and Planets 95, 579-586.

Kero, J., Szasz, C., Nakamura, T., Meisel, D. D., Ueda, M., Fujiwara, Y., Terasawa, T., Miyamoto, H., Nishimura, K., Oct. 2011. First results from the 2009-2010 MU radar head echo observation programme for sporadic and shower meteors: the Orionids 2009. Mon. Not. R. Astron. Soc. 416, 2550-2559.

Mathews, J. D., Janches, D., Meisel, D. D., Zhou, Q.-H., 2001. The micrometeoroid mass flux into the upper atmosphere: Arecibo results and a comparison with prior estimates. Geophys. Res. Lett. 28, 1929-1932.

McNeil, W., 1999. Modeling of Meteor Trails as Observed by LIDAR. In: Jenniskens, P. (Ed.), The Leonid MAC Workshop, NASA Ames Research Center, CA, April 12-15, 1999. Eds.: P. Jenniskens, Meteoritics \& Planetary Science, meeting abstract. 
Michell, R. G., Aug. 2010. Simultaneous optical and radar measurements of meteors using the Poker Flat Incoherent Scatter Radar. Journal of Atmospheric and Solar-Terrestrial Physics 72, 1212-1220.

Moreno-Ibáñez, M., Gritsevich, M., Trigo-Rodríguez, J. M., Apr. 2015. New methodology to determine the terminal height of a fireball. ICARUS 250, $544-552$.

Oppenheim, M. M., vom Endt, A. F., Dyrud, L. P., Oct. 2000. Electrodynamics of meteor trail evolution in the equatorial E-region ionosphere. Geophys. Res. Lett. 27, 3173-3176.

Pifko, S., Janches, D., Close, S., Sparks, J., Nakamura, T., Nesvorny, D., Mar. 2013. The Meteoroid Input Function and predictions of mid-latitude meteor observations by the MU radar. ICARUS 223, 444-459.

Reddi, C. R., Nair, S. M., 1998. Meteor trail induced backscatter in MST radar echoes. Geophys. Res. Lett. 25, 473-476.

Shamir, L., Jun. 2005. Analysis of meteor trails using the Night Sky Live network of panoramic CCD cameras. WGN, Journal of the International Meteor Organization 33, 75-80.

Sparks, J. J., Janches, D., Nicolls, M. J., Heinselman, C., Oct. 2010. Determination of physical and radiant meteor properties using PFISR interferometry measurements of head echoes. Journal of Atmospheric and Solar-Terrestrial Physics 72, 1221-1230.

Stober, G., Jacobi, C., Singer, W., Jun. 2011. Meteoroid mass determination from underdense trails. Journal of Atmospheric and Solar-Terrestrial Physics 73, 895-900.

Von Zahn, U., Nov. 2001. Lidar observations of meteor trails: evidence for fragmentation of meteoroids and their subsequent differential ablation. In: Warmbein, B. (Ed.), Meteoroids 2001 Conference. Vol. 495 of ESA Special Publication. pp. 303-314.

Vondrak, T., Plane, J. M. C., Broadley, S., Janches, D., Dec. 2008. A chemical model of meteoric ablation. Atmospheric Chemistry \& Physics 8, 70157031. 
${ }_{462}$ Weryk, R. J., Brown, P. G., Jun. 2013. Simultaneous radar and video 463 meteors-II: Photometry and ionisation. Planetary and Space Science 81, $464 \quad 32-47$. 